\title{
Markov Partitions and Feigenbaum-like Mappings
}

\author{
Yunping Jiang ${ }^{1}$ \\ Department of Mathematics, Queens College of CUNY, Flushing, NY 11367, USA
}

Received: 4 October 1993/in revised form: 2 September 1994

\begin{abstract}
We construct a Markov partition for a Feigenbaum-like mapping. We prove that this Markov partition has bounded nearby geometry property similar to that for a geometrically finite one-dimensional mappings [8]. Using this property, we give a simple proof that any two Feigenbaum-like mappings are topologically conjugate and the conjugacy is quasisymmetric.
\end{abstract}

\section{Contents}

0 . Introduction

1. Infinitely renormalizable unimodal mappings

2. Markov maps induced from Feigenbaum-like mappings

3. Conjugacies between Feigenbaum-like mappings

\section{Introduction}

Markov process has been introduced by Sinai and Bowen, etc. in the study of dynamical systems in the 1960's. Sinai [16] and Bowen [2] constructed a Markov partition for a hyperbolic dynamical system. Using Markov partitions, they related hyperbolic dynamical systems with symbolic dynamical systems. Thus hyperbolic dynamical systems can be studied topologically through symbolic dynamical systems rather easily. Indeed, to construct a Markov partition for a dynamical system is quite important in the study of dynamical systems. In this note, I shall give a construction of a partition for a Feigenbaum-like mapping. I shall prove that this partition has all but finiteness properties as those of a Markov partition for a hyperbolic dynamical system. It will be called an (infinite) induced Markov partition. A Feigenbaum-like mapping is definitely not hyperbolic for its critical orbit is recurrent. However, from the construction and properties of this induced Markov partition, one can study topologically and geometrically a Feigenbaum-like mapping

\footnotetext{
${ }^{1}$ Partially supported by a PSC-CUNY and a NSF grants
} 
by using methods in hyperbolic dynamical systems. Moreover, using the properties of induced Markov mappings, I give a simple proof that any two Feigenbaum-like mappings are topologically conjugate and the conjugacy is quasisymmetric in this note. The same idea has been successfully used to give a construction of a certain Markov partition of the Julia set of the Feigenbaum quadratic polynomial. This partition enables us to prove a long standing conjecture that the Julia set of the Feigenbaum quadratic polynomial is locally connected. The proof of this theorem and its generalization will be written in a forthcoming paper [9].

This paper is organized as follows: I shall introduce notations and review some known results in Sect. 1. In Sect. 2, I shall construct the induced Markov map from a Feigenbaum-like mapping and prove one of the main results, i.e., this Markov map has bounded nearby geometry. In the last section, I shall apply the property of bounded nearby geometry to prove another main result, i.e., any two Feigenbaumlike mappings are topologically conjugate and the conjugacy between them is quasisymmetric.

\section{Infinitely Renormalizable Unimodal Mappings}

Suppose $I$ is the interval $[-1,1]$. A continuous function $f$ from $I$ into itself is called a unimodal mapping if $f(x)=h\left(-|x|^{\gamma}\right)$ for some real number $\gamma>1$ and some homeomorphism $h$ from $[-1,0]$ onto $[-1, h(0)]$. The Schwarzian derivative $S(g)$ of a $C^{3}$-diffeomorphism $g$ from an interval onto another interval is, by definition,

$$
S(g)=\frac{g^{\prime \prime \prime}}{g^{\prime}}-\frac{3}{2}\left(\frac{g^{\prime \prime}}{g^{\prime}}\right)^{2} .
$$

A unimodal mapping $f(x)=h\left(-|x|^{\gamma}\right)$ is called a $S$-unimodal if $h$ is a $C^{3}$-diffeomorphism from $[-1,0]$ onto $[-1, h(0)]$ and satisfies that $S(h)(x) \leqq 0$ for all $x$ in $[-1,0]$.

A $S$-unimodal mapping $f$ is said to be renormalizable if there is a subinterval $J=[-a, a]$ of $I$ for some $a>0$ and an integer $n \geqq 2$ such that $f^{\circ n}$ are monotone when restricted on $[-a, 0]$ and on $[0, a]$ and $f^{\circ n}(J) \subset J$ and $f^{\circ i}(J) \cap J=$ $\emptyset, 0<i<n$. One can normalize $J$ to $I$ by a linear transformation $\alpha(x)=p x$ such that $\mathscr{R}(f)=\alpha^{-1} \circ f^{\circ n} \circ \alpha$ is a unimodal mapping again (see below). To fix notations, I always assume that $n \geqq 2$ is the smallest such integer and $J$ is the biggest such interval. Thus one can say that $f$ is once $n$-renormalizable and $\mathscr{R}(f)$ is the renormalization of $f$.

Suppose $f$ is a once $n_{1}$-renormalizable $S$-unimodal mapping. If $\mathscr{R}(f)$ is once $n_{2}$-renormalizable, then $f$ is said to be twice $\left(n_{1}, n_{2}\right)$-renormalizable. Further, $f$ is $k$-times $\left(n_{1}, n_{2}, \ldots, n_{k}\right)$-renormalizable if $\mathscr{R}^{\circ i}(f)$ is $n_{i+1}$-renormalizable for $0 \leqq i<$ $k$ and is infinitely $\left(n_{1}, n_{2}, \ldots, n_{k}, \ldots\right)$-renormalizable if $\mathscr{R}^{\circ i}(f)$ is $n_{i+1}$-renormalizable for every integer $i \geqq 0$. A $S$-unimodal mapping $f$ is infinitely $\left(n_{1}, n_{2}, \ldots, n_{k}, \ldots\right)$ renormalizable if and only if there is a sequence $\left\{I_{k}=\left[-a_{k}, a_{k}\right]\right\}_{k=1}^{\infty}$ of nested intervals so that $f^{\circ m_{k}}$ is monotone when restricted on $\left[-a_{k}, 0\right]$ and on $\left[0, a_{k}\right]$, $f^{\circ i}\left(I_{k}\right) \cap I_{k}^{\circ}=\emptyset$ for $0<i<m_{k}$, and $f^{\circ m_{k}}\left(I_{k}\right) \subset I_{k}$, where $m_{k}=\prod_{l=1}^{k} n_{i}$. An infinitely $\left(n_{1}, n_{2}, \ldots, n_{k}, \ldots\right)$-renormalizable $S$-unimodal mapping $f$ is said to be of bounded type if $\left\{n_{k}\right\}_{k=1}^{\infty}$ is a bounded sequence, otherwise $f$ is said to be of unbounded type. In particular, if all $n_{k}=2$, then $f$ is called a Feigenbaum-CoulletTresser-like mapping, in short, Feigenbaum-like mapping [3-5].

Suppose $f(x)=h\left(-|x|^{\gamma}\right)$ is an infinitely $\left(n_{1}, n_{2}, \ldots, n_{k}, \ldots\right)$-renormalizable $S$ unimodal mapping and $m_{k}=\prod_{i=1}^{k} n_{i}$. Let $\mathscr{R}^{\circ k}(f)=\alpha_{k}^{-1} \circ f^{\circ m_{k}} \circ \alpha_{k}$ be the 
$k^{\text {th }}$-renormalization of $f$, where $\alpha_{k}(x)=-p_{k} x$ is the linear rescale from $I=[-1,1]$ to $I_{k}=\left[-a_{k}, a_{k}\right]$, where $a_{k}=\left|p_{k}\right|$ and $I_{k}=\left[-a_{k}, a_{k}\right]$ is the maximal interval containing 0 such that

a. $f^{\circ m_{k}}$ is monotone when restricted on $\left[-a_{k}, 0\right]$ and on $\left[0, a_{k}\right]$,

b. $f^{\circ m_{k}}\left(I_{k}\right) \subset I_{k}$, and $f^{\circ I}\left(I_{k}\right) \cap I_{k}^{\circ}=\emptyset, 0<i<m_{k}$,

c. $f^{\circ m_{k}}$ has exactly two fixed points $p_{k}$ and $q_{k}$ in $I_{k}$ which are also periodic points of $f$ of period $m_{k}$.

Suppose $c(i)=f^{\circ i}(0)$ is the $i^{\text {th }}$ critical value of $f$. For each $k>0$, let $I_{k}(i)=$ $f^{\circ i}\left(I_{k}\right)$ and $p_{k}(i)=f^{\circ i}\left(p_{k}\right)$ be the images of $I_{k}$ and $p_{k}$ under the $i^{\text {th }}$-iterate of $f$. Then $I_{k}(i)$ is an interval bounded by $p_{k}(i)$ and $c(i)$ for $0<i<m_{k}$. Note that $I_{k}(0)=I_{k}$ is an interval bounded by $-p_{k}$ and $p_{k}$ and $I_{k}\left(m_{k}\right)$ is an interval bounded by $p_{k}$ and $c\left(m_{k}\right)$. The mapping $f \mid I_{k}$ is fold from $I_{k}$ onto $I_{k}(1)$ and all other mappings $f \mid I_{k}(i)$ from $I_{k}(i)$ to $I_{k}(i+1)$ are homeomorphisms for $1 \leqq i<m_{k}$ (see Fig. 1). Hence the $k^{\text {th }}$-renormalization can be written into a form $\mathscr{R}^{\circ k}(f)(x)=$ $h_{k}\left(-|x|^{\gamma}\right)$ as a $S$-unimodal mapping, where $h_{k}=\alpha_{k}^{-1} \circ f^{\circ\left(m_{k}-1\right)} \circ h \circ \tilde{\alpha}_{k}$ is a diffeomorphism from $I$ into $h_{k}(I)$, where $\tilde{\alpha}_{k}(x)=\left|p_{k}\right|^{\gamma} x$.

The nonlinearity $N(g)$ of a $C^{2}$-diffeomorphism $g$ from an interval onto another interval is, by definition, $N(g)=g^{\prime \prime} / g^{\prime}$. The a priori real bounds for the nonlinearities of renormalizations of $f$ have been found in [18] (see also [6]). These a priori real bounds depend only on the power law $-|x|^{\gamma}$.

Lemma 1 (Bounded and eventually universally bounded). There is a universal constant $C(\gamma)>0$ and a sequence $\{C(k, \gamma)\}_{k=1}^{+\infty}$ of positive real numbers such that $C(k, \gamma) \rightarrow C(\gamma)$ as $k \rightarrow \infty$ and

$$
\max _{x \in h_{k}([-1,0])}\left|N\left(h_{k}^{-1}\right)(x)\right| \leqq C(k, \gamma) .
$$

Remark 1. For a $C^{2}$-diffeomorphism $h, N\left(h^{-1}\right)(x)=-h^{\prime \prime}(y) /\left(h^{\prime}(y)\right)^{2}$, where $x=$ $h(y)$.

The proof of Lemma 1 can be found in $[12,18]$. The next two lemmas are actually two steps in the proof of Lemma 1. I would like to highlight them. I shall first state a well-known result (see $[12,18$, etc.]) to estimate the nonlinearity of a $C^{3}$-diffeomorphism.

Lemma 2 ( $C^{3}$-Koebe distortion lemma). Suppose $g$ is a $C^{3}$ function on an open interval $J=(a, b)$ and $S(g)(x) \geqq 0$ for all $x$ in $J$. Then

$$
|N(g)(x)| \leqq \frac{2}{d(x, \partial J)}
$$

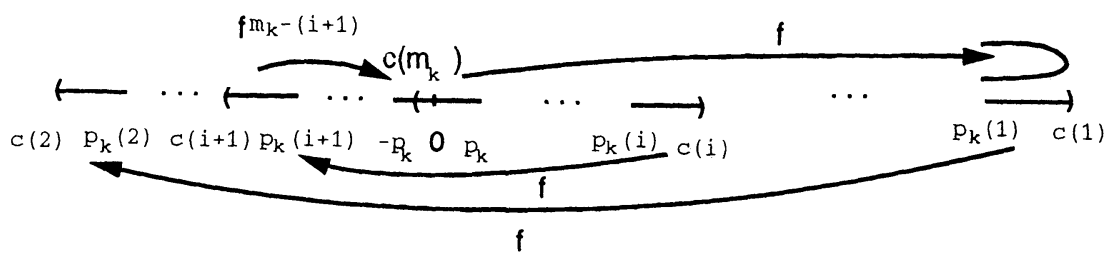

Fig. 1. 


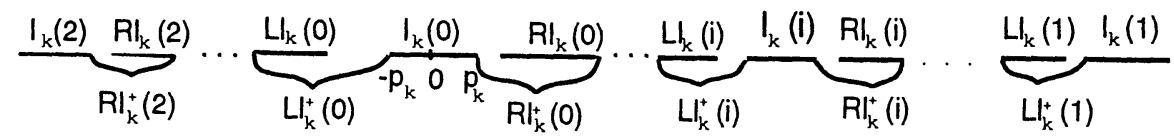

Fig. 2.

for any $x$ in $J$, where $d(x, \partial J)=\min \{|x-a|,|x-b|\}$ is the distance between $x$ and the boundary of $J$.

The second one is about Koebe space around every interval $I_{k}(i)$ (see $[12,18]$ ). Let $\xi_{k}=\left\{I_{k}(i)\right\}_{i=0}^{m_{k}-1}$ for $k=1,2, \ldots$ be the hierarchical system induced from $f$. For each interval $I_{k}(i)$, use $L I_{k}(i)$ and $R I_{k}(i)$ to denote the intervals in $\xi_{k}$ adjacent to $I_{k}(i)$ and in the left and right sides of $I_{k}(i)$, respectively, (there is only $L I_{k}(1)$ or $R I_{k}(2)$ in $\left.\xi_{k}\right)$. Let $L I_{k}^{+}(i)$ be the smallest interval containing $L I_{k}(i)$ and the left end-point of $I_{k}(i)$ and let $R I_{k}^{+}(i)$ be the smallest interval containing $R I_{k}(i)$ and the right end-point of $I_{k}(i)$ for $i=0$ or $3 \leqq i<m_{k}$. Let $L I_{k}^{+}(2)=[-1, c(2)]$ and $R I_{k}^{+}(1)=[c(1), 1]($ see Fig. 2$)$.

Lemma 3. There is a universal constant $C(\gamma)>0$ and a sequence $\{C(k, \gamma)\}_{k=1}^{+\infty}$ of positive real numbers such that $C(k, \gamma) \rightarrow C(\gamma)$ as $k \rightarrow \infty$ and

$$
\min \left\{\left|L I_{k}^{+}(0)\right|,\left|R I_{k}^{+}(0)\right|\right\} \geqq C(k, \gamma)\left|I_{k}(0)\right| \text {. }
$$

\section{Markov Maps Induced From Feigenbaum-like Mappings}

Suppose $f(x)=h\left(-|x|^{\gamma}\right)$ is a Feigenbaum-like mapping. The hierarchical system $\xi_{k}=\left\{I_{k}(i)\right\}_{i=0}^{m_{k}-1}$ for $k=1,2, \ldots$ of $f$ is quite simple. For each $k>0$, the interval $I_{k}(0)$ is bounded by a periodic point $p_{k}$ of $f$ of period $2^{k-1}$ and $-p_{k}$. The mapping $f^{\circ 2^{k}} \mid I_{k}(0)$ has two fixed points $p_{k}$ and $p_{k+1}$. Every interval $I_{k}(i)$ in $\xi_{k}$ contains only two intervals $I_{k+1}(i)$ and $I_{k+1}\left(2^{k}+i\right)$ in $\xi_{k+1}$ which have a common endpoint $p_{k+1}(i)$ for $0 \leqq i<2^{k}$.

Using the sequence of nested intervals $\left\{I_{k}(0)\right\}_{k=1}^{\infty}$, I construct a partition in $I=[-1,1]$. Let $P_{-0}$ and $P_{0}$ be the closures of the left and right connected components of $I \backslash I_{1}(0)$. Inductively, let $P_{-k}$ and $P_{k}$ be the closures of the left and right connected components of $I_{k}(0) \backslash I_{k+1}(0)$. Finally set $P_{\infty}=\{0\}$. The collection $\beta_{0}=$ $\left\{P_{-0}, P_{0}, P_{-1}, P_{1}, \ldots, P_{-k}, P_{k}, \ldots, P_{\infty}\right\}$ forms a partition of $I=[-1,1]$ (see Fig. 3 ), that is, $P_{i}$ and $P_{j}$ have disjoint interiors for $i \neq j$ and $I=P_{\infty} \cup \cup_{k=1}^{\infty}\left(P_{-k} \cup P_{k}\right)$. Let $F$ be the function defined as $F(0)=0$ and

$$
F(x)=\left\{\begin{array}{ll}
f(x), & x \in P_{-0} \cup P_{0} ; \\
f^{\circ 2}(x), & x \in P_{-1} \cup P_{1} ; \\
\vdots & \\
f^{\circ 2^{l}}(x), & x \in P_{-l} \cup P_{i} ; \\
\vdots &
\end{array} .\right.
$$

Then $F$ is continuous on $I$ (see Fig. 3 ). 


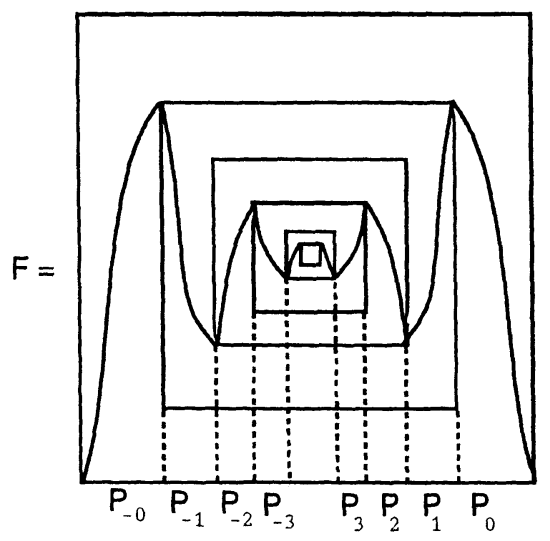

Fig. 3.

Lemma 4. For every even integer $k=2 n \geqq 0, F\left(P_{ \pm k}\right)=\bigcup_{i=k}^{\infty} P_{-i} \cup \bigcup_{i=k+1}^{\infty} P_{i}$, and for every odd integer $k=2 n+1>0, F\left(P_{ \pm k}\right)=\bigcup_{i=k+1}^{\infty} P_{-i} \cup \bigcup_{i=k}^{\infty} P_{i}$.

Proof. It can be seen from Fig. 3.

From Lemma 4, the mapping $F$ and the partition $\beta_{0}$ satisfy the Markov property in the sense that the image of every element in the partition $\beta_{0}$ is the union of some intervals in the partition $\beta_{0}$. Thus I call $F$ the induced Markov mapping from $f$.

Let $g_{ \pm i}=\left(F \mid P_{ \pm i}\right)^{-1}$ be the inverse branches of $F$ for $1 \leqq i<\infty$. Suppose $w=i_{0} i_{1} \cdots i_{k-1}$ is a finite sequence of $\overline{\mathbf{Z}}=\mathbf{Z} \cup\{-0\}$. It is said to be admissible if the range $P_{l_{l}}$ of $g_{i_{l}}$ is contained in the domain $F_{l_{l-1}}\left(P_{i_{l-1}}\right)$ of $g_{i_{l-1}}$ for $l=$ $1, \ldots, k-1$. For an admissible sequence $w=i_{0} i_{1} \cdots i_{k-1}$, define the composition $g_{w}=g_{l_{0}} \circ g_{i_{1}} \circ \cdots \circ g_{i_{k-1}}$. Use $D\left(g_{w}\right)$ to denote the domain of $g_{w}$ and $\left|D\left(g_{w}\right)\right|$ to denote the length of the iterval $D\left(g_{w}\right)$.

Definition 1. We say the induced Markov map $F$ from $f$ has bounded nearby geometry if there is a constant $C=C(f)>0$ such that

(i) $C^{-1} \leqq\left|P_{k}\right| /\left|\bigcup_{i=k+1}^{\infty} P_{i}\right| \leqq C$ and $C^{-1} \leqq\left|P_{-k}\right| /\left|\bigcup_{l=k+1}^{\infty} P_{-i}\right| \leqq C$ for all $k \geqq 0$, and

(ii) $\left|N\left(g_{w}\right)(x)\right| \leqq C /\left|D\left(g_{w}\right)\right|$ for all $x$ in $D\left(g_{w}\right)$ and all finite admissible sequence $w$ of $\bar{Z}$.

Remark 2. Condition (ii) implies that the distortion $\left|\log \left(\left|g_{w}(x)\right| /\left|g_{w}(y)\right|\right)\right|$ of $g_{w}$ at any $x$ and $y$ in $D\left(g_{w}\right)$ is bounded by $C$. Condition (i) is an analogy to bounded nearby geometry defined in [8] for geometrically finite one-dimensional mappings.

Theorem 1. Suppose $f(x)=h\left(-|x|^{\gamma}\right)$ is a Feigenbaum-like S-unimodal mapping. Then the induced Markov mapping $F$ from $f$ has bounded nearby geometry. 
Before proving Theorem 1, I shall prove some useful lemmas.

Lemma 5. Suppose $h$ from $[-1,0]$ to $\mathbf{R}^{1}$ is a $C^{3}$ orientation-preserving diffeomorphism and $S(h)(x) \leqq 0$ for all $x$ in $[-1,0]$. Suppose $\phi$ is a linear fractional transformation satisfying that

(a) $\phi(a)=h(a)$ for $a=0$ and -1 , and

(b) $N\left(h^{-1}\right)(-1) \geqq N\left(\phi^{-1}\right)(-1)$.

Then $\phi(x) \leqq h(x)$ for all $x$ in $[-1,0]$.

Proof. Let $Z=h^{-1} \circ \phi$. Then $Z(a)=a$ for $a=0$ and -1 and for $x$ in $[-1,0]$,

$$
S(Z)(x)=\left(\phi^{\prime}(x)\right)^{2} \cdot\left(S\left(h^{-1}\right)\right)(\phi(x)) \geqq 0 .
$$

The goal is to show that $Z(x) \leqq x$ for $x$ in $[-1,0]$.

Using (b), one can get $N(Z)(-1) \geqq 0$. This implies that $Z^{\prime \prime}(-1) \geqq 0$, and moreover,

$$
Z(x) \geqq F(x)=-1+Z^{\prime}(-1)(x+1)
$$

for small $x+1 \geqq 0$. Thus $Z(x) \geqq F(x)$ for all $x$ in $[-1,0]$ since $S(Z)(x) \geqq 0$ for all $x$ in $[-1,0]$. In particular, $Z(0) \geqq F(0)$. Hence $Z^{\prime}(-1) \leqq 1$. Therefore, $Z(x) \leqq$ $x$ for all $x$ in $[-1,0]$ because $S(Z)(x) \geqq 0$ for all $x$ in $[-1,0]$. So $\phi(x) \leqq h(x)$ for all $x$ in $[-1,0]$.

Let $S F(\gamma, C)$ be the subspace of $S$-unimodal mappings $f(x)=h\left(-|x|^{\gamma}\right)$ so that $\min _{x \in h([-1,0])}\left(N\left(h^{-1}\right)(x)\right) \geqq-C$.

Lemma 6. There is a constant $C_{1}=C_{1}(\gamma, C)>0$ such that $f(0) \geqq C_{1}$ for every infinitely renormalizable mapping in $S F(\gamma, C)$.

Proof. Suppose $f(x)=h\left(-|x|^{\gamma}\right)$ is a mapping in $S F(\gamma, C)$. Since $h$ is a $C^{3}$ orientation-preserving diffeomorphism and $S(h)(x) \leqq 0$ for all $x$ in $[-1,0]$, one can compare $h$ with some linear fractional transformation $\phi(x)=(a x+b) /(c x+d)$. Let $\phi$ be the linear fractional trnsformation satisfying that (a) $\phi(a)=h(a)$ for $a=-1$ and 0 , and (b) $N\left(\phi^{-1}\right)(-1)=-C$. Then

$$
\phi(x)=\frac{x+1}{\frac{C}{2}(x+1)+\frac{1}{f(0)+1}-\frac{C}{2}}-1 .
$$

From Lemma $5, \phi(x) \leqq h(x)$ for all $x$ in $[-1,0]$.

Suppose, at the moment, $c=f(0)>0$ is a variable. Let $C_{1}=C_{1}(\gamma, C)>0$ be the smallest solution of $\phi\left(-|c|^{\gamma}\right)=0$. Then for $0<c<C_{1}, f^{\circ 2}(0) \geqq \phi^{\circ 2}(0)=$ $\phi\left(-|c|^{\gamma}\right)>0$. This says that $f^{\circ 2}$ has an attractive fixed point and thus is not once renormalizable. Hence $f(0)>C_{1}$ if $f$ is infinitely renormalizable.

For a $S$-unimodal mapping $f(x)=h\left(-|x|^{\gamma}\right)$ with $f(0)>0$, let $q_{f}$ be the fixed point of $f$ in $(0,1)$.

Lemma 7. There is a constant $C_{2}=C_{2}(\gamma, C)>0$ such that $q_{f} \geqq C_{2}$ for all infinitely renormalizable $f$ is $S F(\gamma, C)$.

Proof. Let

$$
\phi_{0}(x)=\frac{x+1}{\frac{C}{2}(x+1)+\frac{1}{C_{1}+1}-\frac{C}{2}}-1
$$


and $C_{2}$ be the fixed point of $\phi_{0}\left(-|x|^{\gamma}\right)$ in $(0,1)$. Take

$$
\phi(x)=\frac{x+1}{\frac{C}{2}(x+1)+\frac{1}{f(0)+1}-\frac{C}{2}}-1 .
$$

Then the fixed point $q^{\prime}$ of $\phi\left(-|x|^{\gamma}\right)$ in $(0,1)$ is greater than $C_{2}$ since $f(0)>C_{1}$. But $q_{f} \geqq q^{\prime}>C_{2}$.

Proof of Theorem 1. Suppose $\mathscr{R}^{\circ k}(f)=h_{k}\left(-|x|^{\gamma}\right)$ is the $k^{\text {th }}$-renormalization of $f$. It is the rescale of $f^{\circ 2^{k}} \mid I_{k}(0)$. From Lemma 1 , there is a constant $C=C(f)>$ 0 such that $\max _{x \in h_{k}([-1,0])}\left|N\left(h_{k}^{-1}\right)(x)\right| \leqq C$ for all $k \geqq 0$ (set $h_{0}=h$ ). Now Lemma 7 says that there is a constant $C_{2}=C_{2}(\gamma, C)>0$ so that

$$
\frac{\left|I_{k+1}(0)\right|}{\left|I_{k}(0)\right|} \geqq C_{2}
$$

for all $k \geqq 0\left(\right.$ set $\left.I_{0}(0)=I\right)$.

Following Lemma 3 , there is a constant $C_{3}=C_{3}(f)>0$ such that

$$
\frac{\left|I_{k+1}\left(2^{k}\right)\right|}{\left|I_{k+1}(0)\right|} \geqq C_{3}
$$

for all $k \geqq 0$ since $I_{k+1}\left(2^{k}\right)$ is either $L I_{k+1}^{+}(0)$ or $R I_{k+1}^{+}(0)$. This implies that

$$
\frac{\left|I_{k+1}(0)\right|}{\left|I_{k}(0)\right|} \leqq C_{4}=\frac{1}{2 C_{3}+1}
$$

for all $k \geqq 0$.

Now take $C_{5}=\max \left\{C_{2}^{-1}, C_{4}\right\}$. Then

$$
C_{5}^{-1} \leqq \frac{\left|I_{k+1}(0)\right|}{\left|I_{k}(0)\right|} \leqq C_{5}
$$

for all $k \geqq 0$.

Since $I_{k}(0) \backslash I_{k+1}(0)$ is the closure of $\bigcup_{i=k+1}^{\infty}\left(P_{-i} \cup P_{i}\right)$, and $\left|P_{-k}\right|=\left|P_{k}\right|$, (i) of Definition 1 is verified.

Now let me prove (ii) of Definition 1. For an integer $i \neq 0, g_{i}$ can be extended to the interval $\Omega_{|i|}=I_{|i|-1}\left(2^{|i|-1}\right) \cup D\left(g_{i}\right) \cup I_{|i|}\left(2^{|i|}\right)$ as a $C^{3}$-diffeomorphism and $S\left(g_{i}\right)(x) \geqq 0$ for all $x$ in $\Omega_{|i|}$. For $g_{0}$ and $g_{-0}$, without loss of generality, we may assume that they can be extended to the interval $\Omega_{0}=(-\infty,-1] \cup D\left(g_{0}\right) \cup I_{1}(1)$ and $S\left(g_{0}\right)(x) \geqq 0$ and $S\left(g_{0}\right)(x) \geqq 0$ for all $x$ in $\Omega_{0}$.

Suppose $w=i_{0} i_{1} \cdots i_{k-1}$ is an admissible sequence of $\bar{Z}=\mathbf{Z} \cup\{-0\}$ and $g_{w}=g_{i_{0}} \circ g_{i_{1}} \circ \cdots \circ g_{i_{k-1}}$. By the definition of an admissible sequence, one can check that

$$
\left|i_{0}\right| \leqq\left|i_{i}\right| \leqq \cdots \leqq\left|i_{k-1}\right|
$$

Hence $g_{w}$ can be extended to the domain $\Omega_{\left|l_{k-1}\right|}$ as a $C^{3}$-diffeomorphism and $S\left(g_{w}\right)(x) \geqq 0$ for all $x$ in $\Omega_{\left|i_{k-1}\right|}$. We note that $D\left(g_{w}\right)=D\left(g_{i_{k-1}}\right)$ and the intervals $\Omega_{|i|}$ are nested for $|i|=0,1, \ldots$. Then (ii) of Definition 1 follows now from Lemmas 2 and 3.

Remark 3. In [10], there is a more general discussion of the induced Markov map from an infinitely renormalizable $S$-unimodal mapping. 


\section{Conjugacies Between Feigenbaum-like Mappings}

It is known that two Feigenbaum-like $S$-unimodal mappings are topologically conjugate. The proof of this depends on two deep facts, kneading theory developed by Milnor and Thurston [14] and non-wandering interval theorem proved by Guckenheimer [7] and de Melo and van Strien [13]. Using Markov partitions constructed in this note, I can set a topological model for all Feigenbaum-like mappings as Sinai and Bowen $[2,16]$ did for hyperbolic dynamical systems. Applying Theorem 1, I give a simple proof of that two Feigenbaum-like $S$-unimodal mappings are topologically conjugate.

Suppose $f$ is a Feigenbaum-like mapping and $\beta_{0}=\left\{P_{ \pm k}\right\}_{k=0}^{\infty} \cup\left\{P_{\infty}\right\}$ is the induced partition and $F$ is the induced Markov map. Let $A=\left(a_{i j}\right)$ be the biinfinite matrix so that $a_{i j}=1$ if $P_{j} \subset F\left(P_{l}\right)$ and $a_{i j}=0$ otherwise. From the construction of $F$, we can see that 1 . for $i= \pm 2 n, n \geqq 0, a_{i j}=1$ if and only if $|j|>|i|$ or $j=-2 n ; \mathbf{2} . i= \pm(2 n+1)>0, n>0, a_{i j}=1$ if and only if $|j|>|i|$ or $j=2 n+1$. Now consider the symbolic space $\Sigma_{A}=\left\{w=i_{0} i_{1} \cdots i_{k} i_{k+1} \cdots \mid i_{k} \in\right.$ $\left.\bar{Z} \cup\{\infty\}, a_{i_{k} i_{k+1}}=1, k=0,1, \ldots\right\}$ with product topology and the shift map $\sigma_{A}(w)=$ $i_{1} \cdots i_{k} i_{k+1} \cdots$ if $w=i_{0} i_{1} \cdots i_{k} i_{k+1} \cdots$.

A sequence $w_{k}=i_{0} i_{1} \cdots i_{k}$ of $\overline{\mathbf{Z}}$ is admissible if $a_{i_{l} i_{l+1}}=1$ for $0 \leqq l<k$. For an admissible sequence $w_{k}=i_{0} i_{1} \cdots i_{k}$, define $g_{w_{k}}=g_{l_{0}} \circ g_{i_{1}} \circ \cdots \circ g_{i_{k}}$ and $P_{w_{k}}=g_{w_{k}}\left(F\left(P_{i_{k}}\right)\right)$.

Lemma 8. Suppose $f$ is a Feigenbaum-like S-unimodal mapping and $F$ is the induced Markov map. Then $F$ is semi-conjugate to $\sigma_{A}$, this means that there is a continuous surjective map $H$ from $\Sigma_{A}$ to I such that $F \circ H=H \circ \sigma_{A}$.

Proof. For any $w=i_{0} i_{1} \cdots i_{k} i_{k+1} \cdots$ in $\Sigma_{A}, w_{k}=i_{0} \cdots i_{k}$ is admissible for every $k \geqq 0$. Applying Theorem $1, \bigcap_{k=0}^{\infty} P_{w_{k}}$ contains only one point $x_{w}$. Set $H(w)=x_{w}$. It is a continuous map from $\Sigma_{A}$ to $I$ from Theorem 1. Since $\bigcup_{w_{k}} P_{w_{k}}=I$, where $w_{k}$ runs over all admissible sequences of length $k+1, H$ is surjective. Moreover, every point $x$ has at most two preimages in $\Sigma_{A}$ under $H$ and only a boundary point $x$ of $P_{w_{k}}$ for some admissible $w_{k}$ has two preimages. Now it is easy to see $F \circ H(w)=H \circ \sigma_{A}(w)$.

Theorem 2. Any two Feigenbaum-like mappings $f$ and $g$ are topologically conjugate.

Proof. Suppose $F$ and $G$ are the induced Markov maps from $f$ and $g$. Let $H_{1}$ and $H_{2}$ be the semi-conjugacies from $F$ and $G$ to $\sigma_{A}$. From the proof of Lemma 8, $H=H_{1} \circ H_{2}^{-1}$ can be defined as a homeomorphism of $I$ and $F \circ H=H \circ G$. Hence $F$ and $G$ are topologically conjugate. Furthermore, $H$ is also the conjugacy between $f$ and $g$.

A homeomorphism $H$ of $I=[-1,1]$ is said to be quasisymmetric [1] if there is a constant $C>0$ so that for any $x$ and $y$ in $I$,

$$
C^{-1} \leqq \frac{|H(x)-H(z)|}{|H(z)-H(y)|} \leqq C,
$$

where $z=(x+y) / 2$. Furthermore, I can use a similar method to that in [8] to prove

Theorem 3. Suppose $f$ and $g$ are two Feignbaum-like mappings and $H$ is the conjugacy between them. Then $H$ is quasisymmetric. 
Proof. Suppose $\beta_{0, f}=\left\{P_{ \pm k, f}\right\}_{k=0}^{\infty} \cup\left\{P_{\infty, f}\right\}$ is the induced Markov partition from $f$ and $\beta_{0, g}=\left\{P_{ \pm k, g}\right\}_{k=0}^{\infty} \cup\left\{P_{\infty, g}\right\}$ is the induced Markov partition from $g$. Let $\beta_{k, f}=\left\{P_{w_{k}, f} \mid w_{k}\right.$ be an admissible sequence of $\bar{Z} \cup\{\infty\}$ of length $\left.k+1\right\}$ and $\beta_{k, g}=\left\{P_{w_{k}, g} \mid w_{k}\right.$ is an admissible sequence of $\bar{Z} \cup\{\infty\}$ of length $\left.k+1\right\}$. They are called induced $k^{\text {th }}$-partitions of $I=[-1,1]$ from $f$ and $g$. From Theorem 1 , there is a constant $C>0$ so that

$$
C^{-1} \leqq \frac{\left|P_{ \pm l w_{k}, f}\right|}{\left|\bigcup_{i=l+1}^{\infty} P_{ \pm i w_{k}, f}\right|} \leqq C
$$

and

$$
C^{-1} \leqq \frac{\left|P_{ \pm l w_{k}, g}\right|}{\left|\bigcup_{l=l+1}^{\infty} P_{ \pm i w_{k}, g}\right|} \leqq C
$$

for all $l$ in $\bar{Z}$ and all admissible sequences $w_{k}$ of $\bar{Z}$ of length $k+1$. This exactly means that the hierarchical system $\left\{\beta_{k, f}\right\}_{k=0}^{\infty}$ and $\left\{\beta_{k, g}\right\}_{k=0}^{\infty}$ satisfy a similar property to bounded nearby geometry defined in [8] for a geometrically finite one-dimensional mapping. Now using a similar argument to the proof of Theorem B in [8], one can prove that $H$ is quasisymmetric. However, for the sake of completeness of this note, I shall write down the proof in more details.

I first construct a little different sequence of nested partitions $\left\{\eta_{k, f}\right\}_{k=0}^{\infty}$ of $I=$ $[-1,1]$ from $\beta_{0, f}$. Let $\eta_{0, f}$ consist of one interval $I$. Cut $I$ into three intervals $L_{0}=P_{-0, f}, M_{0}=c l\left(\bigcup_{i=1}^{\infty}\left(P_{-i, f} \cup P_{l, f}\right)\right)$, where $c l$ means closure, and $R_{0}=P_{0, f}$ (see Fig. 4). Then $\eta_{1, f}=\left\{L_{0}, M_{0}, R_{0}\right\}$.

The map $F$ is a diffeomorphism when restricted on $L_{0}$ or $R_{0}$ and $F\left(L_{0}\right)=$ $F\left(R_{0}\right)=L_{0} \cup M_{0}$. Cut $L_{0}$ (respectively, $R_{0}$ ) into two intervals $L_{0} L_{0}$ and $L_{0} M_{0}$ (respectively, $R_{0} L_{0}$ and $R_{0} M_{0}$ ) which are preimages of $L_{0}$ and $M_{0}$ under $F \mid L_{0}$ (respectively, $F \mid R_{0}$ ). And cut $M_{0}$ into three intervals $L_{1}=P_{-1, f}, M_{1}=$ $c l\left(\bigcup_{i=2}^{\infty}\left(P_{-l, f} \cup P_{i, f}\right)\right)$, and $R_{1}=P_{1, f}$ (see Fig. 5). Then

$$
\eta_{2, f}=\left\{L_{0} L_{0}, L_{0} M_{0}, L_{1}, M_{1}, R_{1}, R_{0} L_{0}, R_{0} M_{0}\right\}
$$

Now I shall define $\eta_{n, f}$ for $n \geqq 3$ inductively. Suppose $\eta_{n, f}$ has been defined for some $n \geqq 2$ and contains $L_{n-1}=P_{-(n-1), f}, M_{n-1}=c l\left(\bigcup_{i=n}^{\infty}\left(P_{-l, f} \cup P_{l, f}\right)\right)$, and $\quad R_{n-1}=P_{n-1, f}$. Cut $M_{n-1}$ into three intervals $L_{n}=P_{-n, f}, \quad M_{n}=$ $c l\left(\bigcup_{i=n+1}^{\infty}\left(P_{-i, f} \cup P_{l, f}\right)\right)$, and $R_{n}=P_{n, f}$. For an interval $J \neq M_{n-1}$ in $\eta_{n, f}$, there

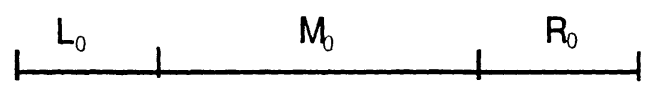

Fig. 4.

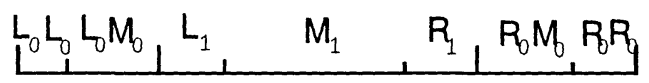

Fig. 5. 
is the maximum integer $i \geqq 1$ such that $F^{\circ i} \mid J$ is a diffeomorphism. Then $F^{\circ i}(J)$ is either 1) $M_{n-i-1}$ or 2) $L_{n-i} \cup M_{n-i}$ or 3) $R_{n-i} \cup M_{n-i}$. In case 1), cut $J$ into three intervals $\mathscr{J}=\left\{J L_{n-i}, J M_{n-i}, J R_{n-i}\right\}$ which are the preimages of $L_{n-i}, M_{n-i}$ and $R_{n-i}$ under $F^{\circ i} \mid J$. In case 2), cut $J$ into two intervals $\mathscr{J}=\left\{J L_{n-i}, J M_{n-i}\right\}$ which are the preimages of $L_{n-i}$ and $M_{n-i}$ under $F^{\circ i} \mid J$. In case 3), cut $J$ into two intervals $\mathscr{J}=\left\{J M_{n-i}, J R_{n-i},\right\}$ which are the preimages of $M_{n-i}$ and $R_{n-i}$ under $F^{\circ i} \mid J$. Then

$$
\eta_{n+1, f}=\left\{\mathscr{J} \mid J \in \eta_{n, f}\right\} \cup\left\{L_{n}, M_{n}, R_{n}\right\} .
$$

Therefore I have defined a sequence $\eta_{f}=\left\{\eta_{n, f}\right\}_{n=0}^{\infty}$ of nested partitions from $\beta_{0, f}$. Similarly, one can define a sequence $\eta_{g}=\left\{\eta_{n, g}\right\}_{n=0}^{\infty}$ of nested partitions from $\beta_{0, g}$.

From the construction of $\eta_{f}$ and Theorem $1, \eta_{f}$ has bounded and bounded nearby geometry which is defined in [8]. More precisely, there is a constant $C>0$ such that (BG) (bounded geometry): for any intervals $J \subset T$ with $J$ in $\eta_{n+1}$ and $T$ in $\eta_{n}, n \geqq 0,|J| /|T| \geqq C$, and (BNG) (bounded nearby geometry): for any intervals $J_{1}$ and $J_{2}$ in $\eta_{n}$ with a common endpoint, $n \geqq 1,\left|J_{1}\right| /\left|J_{2}\right| \geqq C$.

The statement (BG) follows from Theorem 1 and the construction of $\eta_{f}$ directly. To prove the statement (BNG), one need to check when the common endpoint point $q$ of $J_{1}$ and $J_{2}$ is a preimage of a fixed point $p_{k}$ of $F$ under $F$. In this case, let $J_{1, i}=F^{\circ i}\left(J_{1}\right)$ and $J_{2, i}=F^{\circ i}\left(J_{2}\right)$ for $i \geqq 0$. Then there is the biggest integer $j \geqq 0$ such that $F^{\circ j} \mid J_{1} \cup J_{2}$ is a diffeomorphism. So $F^{\circ j}(q)=p_{ \pm k}$. Therefore there exists another integer $m \geqq j$ such that both of $F^{\circ m} \mid J_{1}$ and $F^{\circ(m+1)} \mid J_{2}$ are diffeomorphisms and $J_{1, m}=J_{2, m+1}=P_{k}$ or $P_{-k}$. This implies that $J_{1, l}=J_{2, l+1}$ for all $j<l \leqq m$. In particular, $J_{1, j}=J_{2, j+1}$. So $J_{1, j}=F\left(J_{2, j}\right)$. From Theorem 1 , there is a constant $C_{0}>0$ such that $C_{0}^{-1} \leqq F^{\prime}(x) \leqq C_{0}$ for all $x$ in $P_{ \pm k}$ and $k \geqq 0$. Hence

$$
C_{0}^{-1} \leqq \frac{\left|J_{1, j}\right|}{\left|J_{2, j}\right|} \leqq C_{0}
$$

Applying Theorem 1 again, there is a constant $C_{1}>0$ such that

$$
C_{1}^{-1} \leqq \frac{\left|J_{1}\right|}{\left|J_{2}\right|} \leqq C_{1}
$$

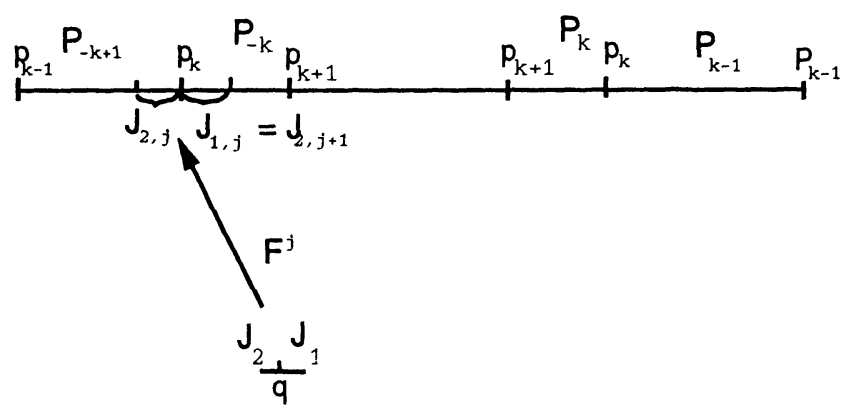

Fig. 6. 
Similarly, one can prove that $\eta_{g}$ has bounded and bounded nearby geometry $((\mathbf{B G})$ and (BNG)) too.

Now I use the property of bounded and bounded nearby geometry $((\mathbf{B G})$ and (BNG)) of $\eta_{f}$ and $\eta_{g}$ to prove that $h$ is quasisymmetric (refer to [8]). For any $x<y$ in $I$, let $z=(x+y) / 2$ be the midpoint between $x$ and $y$ and $N>0$ be the smallest integer such that there is an interval $J$ in $\eta_{K, f}$ contained in $[x, y]$. Let $\tilde{J}$ be the interval in $\eta_{N-1, f}$ containing $J$. Then the union of $\tilde{J}$ and one $J J$ of its adjacent intervals in $\eta_{N-1, f}$ contains $[x, y]$ (see Figs. 7,8 and 9). Because of bounded and bounded nearby geometry ((BG) and (BNG)) of $\eta_{g}$ (and refer to Figs. 7,8 and 9), there is a constant $C_{2}>0$ such that

$$
\frac{|H(J)|}{|H([x, z])|} \geqq C_{2} \quad \text { and } \quad \frac{|H(J)|}{|H([z, y])|} \geqq C_{2} .
$$

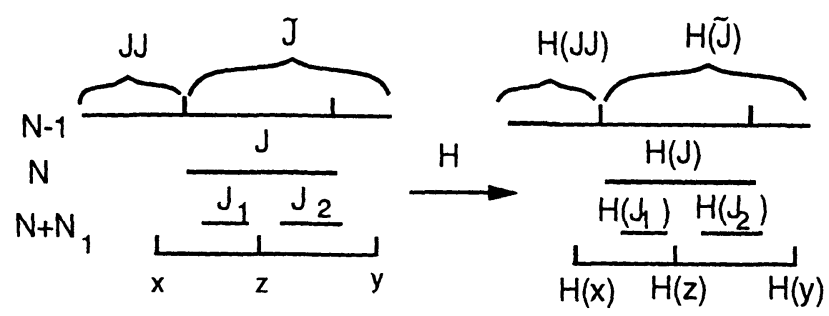

Fig. 7.
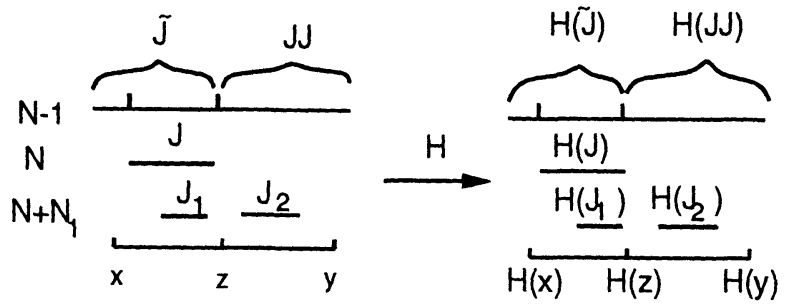

Fig. 8.
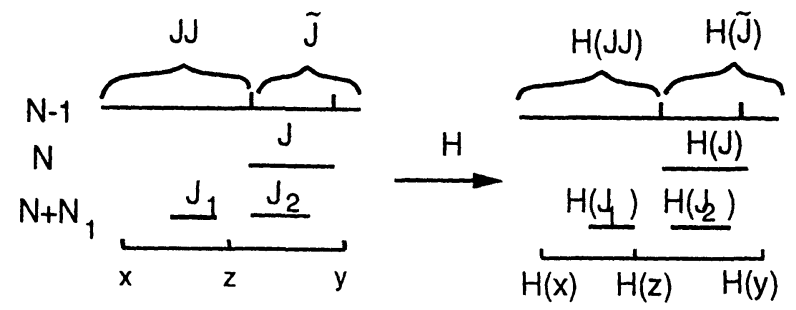

Fig. 9. 
Since $\eta_{f}$ has bounded geometry (BG), the maximum length of intervals in $\eta_{n, f}$ tends to zero exponentially, that is, there is a constant $C_{3}>0$ and $0<\lambda<1$ such that

$$
\max _{J \in \eta_{n, f}}|J| \leqq C_{3} \lambda^{n}
$$

for all $n \geqq 0$. Thus there is a constant integer $N_{1}>0$ (does not depend on $N$ ) such that there are intervals $J_{1}$ and $J_{2}$ in $\eta_{N+N_{1}}$ contained in $[x, z]$ and $[z, y]$, respectively. This implies that $H\left(J_{1}\right)$ and $H\left(J_{2}\right)$ are contained in $H([x, z])$ and $H([z, y])$ respectively because $H$ is the conjugacy.

Because of bounded and bounded nearby geometry ((BG) and (BNG)) of $\eta_{g}$ again, there is a constant $C_{4}>0$ (see Figs. 7,8 and 9) such that

$$
C_{4}^{-1} \leqq \frac{|H(x)-H(z)|}{|H(z)-H(y)|} \leqq C_{4}
$$

which means that $H$ is quasisymmetric.

Remark 5. The quasisymmetric property of a conjugacy is first studied in [18] for complex quadratic-like Feigenbaum-like mappings by using the complex method. The proof of Theorem 3 here is for more general unimodal mappings and is a real method developed from $[8,10]$. A different approach to the proof in the general case was tried in [15]. This theorem can be also proven for infinitely renormalizable $S$-unimodal mappings of bounded type (see, for example, [10]). However, for infinitely renormalizable $S$-unimodal mappings of unbounded type, it is still an open question. In $[11,17]$, a result about infinitely renormalizable quadratic polynomials of unbounded type has been announced recently.

Acknowledgement. I would like to thank the referee for valuable suggestions.

\section{References}

1. Ahlfors, L.: Lectures on Quasiconformal Mappings. Princeton, NJ: Van Nostrand-Reinhold, 1966

2. Bowen, R.: Equilibrium States and the Ergodic Theory of Anosov Diffeomorphisms. Lecture Notes in Mathematics, Vol. 470, Berlin, Heidelberg, New York: Springer, 1975

3. Coullet, P., Tresser, C.: Itération d'endomorphismes et groupe de renormalisation. C.R. Acad. Sci. Paris Ser., A-B 287, A577-A580 (1978)

4. Feigenbaum, M.: Quantitative universality for a class of non-linear transformation. J. Stat. Phys. 19, 25-52 (1978)

5. Feigenbaum, M.: The universal metric properties of non-linear transformation. J. Stat. Phys 21, 669-706 (1979)

6. Guckenheimer, J.: Limit sets of $S$-unimodal maps with zero entropy. Commun. Math. Phys. 110, 655-659 (1987)

7. Guckenheimer, J.: Sensitive dependence on initial conditions for one-dimensional maps. Commun. Math. Phys. 70, 133-160 (1979)

8. Jiang, Y.: Geometry of geometrically finite one-dimensional mappings. Commun. Math. Phys. 156, 639-647 (1993)

9. Jiang, Y.: Infinitely renormalizable quadratic Julia sets. Research Report of FIM, ETH, Zürich, June, 1994

10. Jiang, Y.: On quasisymmetrical classification of infinitely renormalizable maps - I. Maps with Feigenbaum topology. and II. Remarks on maps with a bounded type topology. IMS preprint series 1991/199, SUNY at Stony Brook, 1991 
11. Lyubich, M.: Geometry of quadratic polynomials: Moduli, rigidity, and local connectivity. IMS preprint series, 1993/9, SUNY@Stony Brook

12. de Melo, W., van Strien, S.: One-dimensional Dynamics. Berlin, Heidelberg, New York: Springer, 1993

13. de Melo, W., van Strien, S.: A structure theorem in one dimensional dynamics. Ann. Math. 129, 519-546 (1989)

14. Milnor, J., Thurston, W.: On iterated maps of the interval: I and II. In: Lecture Notes in Mathematics., Vol. 1342, Berlin, Heidelberg, New York: Springer, 1988, pp. 465-563

15. Paluba, W.: Talks in Graduate Center of CUNY, 1991 and 1992

16. Sinai, Ya.: Markov partitions and $C$-diffeomorphisms. Funct. Anal. and its Appl. 2, no. 1, 64-89 (1968)

17. Swiatek, G.: Hyperbolicity is dense in real quadratic family. IMS preprint series 1992/9, SUNY@StonyBrook, 1992

18. Sullivan, D.: Bounds, quadratic differentials, and renormalization conjectures. American Mathematical Society Centennial Publications, Volume 2: Mathematics into the Twenty-first Century, Providence, RI: AMS, 1991

Communicated by J.-P. Eckmann 
